\title{
Hope for axed cancer-prevention trial
}

Researchers behind a major trial on breast-cancer prevention whose funding was cancelled in June say that Elias Zerhouni, director of the US National Institutes of Health (NIH) has indicated that he may be willing to meet them.

The trial was to have received US\$55 million from the National Cancer Institute (NCI). It would have involved almost 13,000 postmenopausal women at a high risk of developing breast cancer, and aimed to investigate the efficacy of two cancer drugs - letrozole and raloxifene - in preventing the disease. It was initially approved by NCI staff, external reviewers and, in January, by an executive committee of senior NCI leaders. But the next day, NCI director John Niederhuber put the funding on hold. He convened an ad hoc committee to study the costly trial further.

Niederhuber later cancelled the funding, partly over fears of giving healthy women pills with known side effects and the public's reluctance to take preventative medicines. Previous

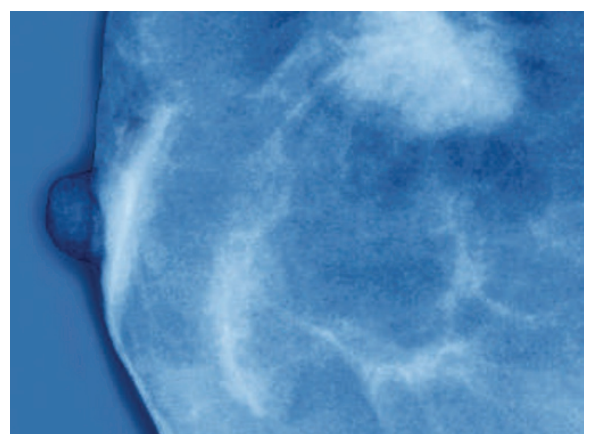

Drugs to prevent breast cancer: worth the cost?

studies, he wrote, "have failed to change the practice of breast cancer prevention among women and their [doctors], perhaps because of the side effects of these drugs".

The scientists leading the trial at the National Surgical Adjuvant Breast and Bowel Project, based in Pittsburgh, Pennsylvania, protested vehemently, and accused Niederhuber of "acting unilaterally". On 20 July, they hand-delivered a 13-page appeal to Zerhouni, asking him to intervene. Last week they said, "we've had some very positive interactions" with Zerhouni's office, and they are hopeful of meeting him this month.

The appeal has drawn media attention in the United States, and in a recent editorial The Lancet labelled Niederhuber's actions "troubling" and called for an investigation (Lancet 369, 2134; 2007).

But Bruce Chabner of Massachusetts General Hospital, who chaired Niederhuber's ad hoc committee, says that the NIH's frozen budget has forced Niederhuber to make tough fiscal choices. He adds that the panel was strongly influenced by the fact that letrozole, made by Novartis, goes off-patent in 2011. "Was it worth spending millions on a trial that might never result in a drug being used for this indication?" he asks. "We thought it likely it wasn't."

Meredith Wadman 